\title{
Tandem Photodetectors Containing Silicon Nanowires with Selective Spectral Absorption
}

\author{
Hyunsung Park ${ }^{1}$ and Kenneth B. Crozier, $1,2,3, *$ \\ ${ }^{1}$ School of Engineering and Applied Sciences, Harvard University, Cambridge, MA 02138, USA \\ ${ }^{2}$ Department of Electrical and Electronic Engineering, University of Melbourne, VIC 3010, Australia \\ ${ }^{3}$ School of Physics, University of Melbourne, VIC 3010, Australia \\ *kcrozier@unimelb.edu.au
}

\begin{abstract}
We fabricate a vertically stacked photodetector device containing silicon nanowire photodetectors formed above a silicon substrate that also contains a photodetector. The substrate photodetector converts light not absorbed by the nanowires to photocurrent.

OCIS codes: (040.5160) Photodetectors; (220.4241) Nanostructure fabrication
\end{abstract}

Optoelectronic devices based on semiconductor nanowires (NWs) are attracting much interest, due to potential applications that could arise from the fact that NWs have optical properties that differ from those of bulk materials. It has been shown that horizontal germanium NWs have absorption spectra that vary with radius [1]. We demonstrated that etched vertical silicon NWs exhibit colors that depend on their radii due to wavelength-selective coupling and absorption [2]. We also developed color image sensor pixels comprising vertical silicon NWs incorporating axial p-i-n junctions and performed color imaging without the need for additional color filters [3]. In that work however, the light not absorbed by the NWs was transmitted into the substrate and not detected [3]. Here, we demonstrate a tandem device consisting of a NW array photodetector formed above a substrate containing a photodetector. Part of the spectrum is captured by the NW photodetector and the remainder is captured by the substrate photodetector (Fig. 1(a)). In a conventional image sensor, color separations are performed by absorptive color filters. These are inherently inefficient in that they only transmit certain spectral bands, with incident photons of other energies being discarded. On the other hand, in the device we demonstrate, the NW array acts as a filter, but with the unusual property that absorbed photons are converted to photocurrent. This presents the opportunity for image sensor pixels for color or multispectral imaging with very high photon efficiencies.
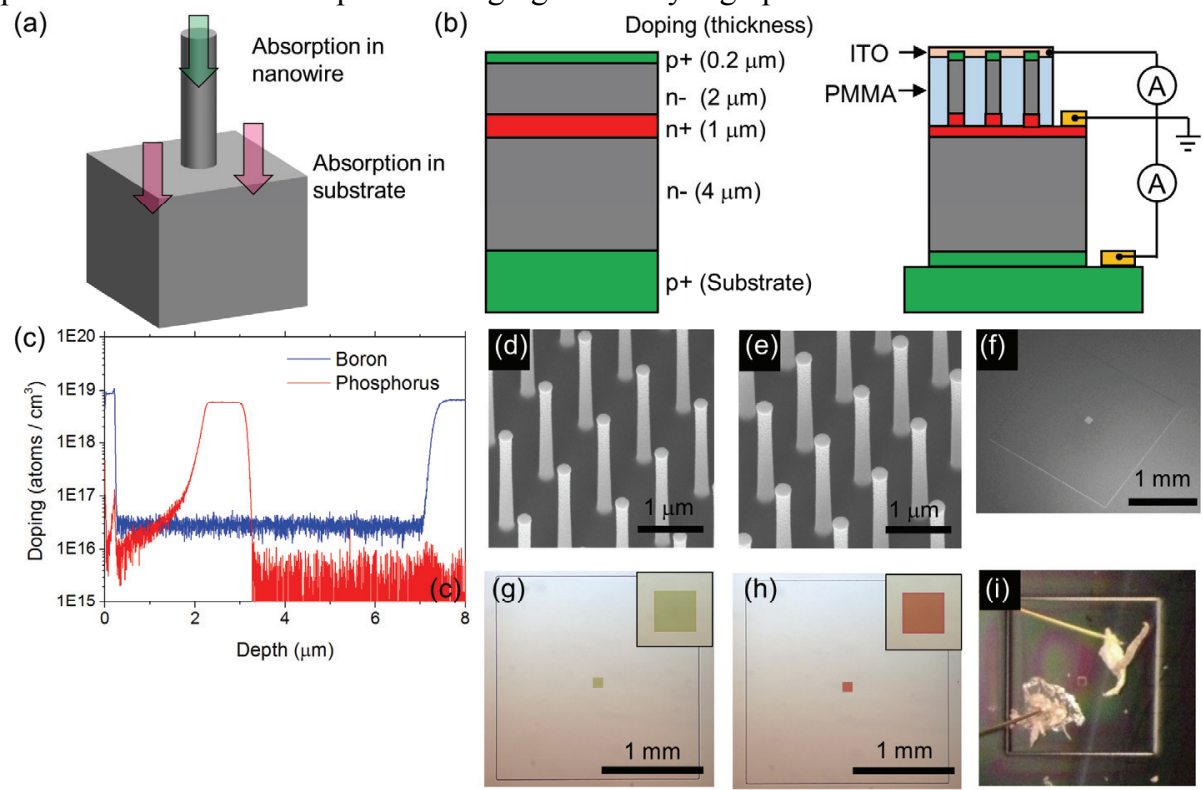

Figure 1 (a) Schematic illustration of vertically stacked tandem photodetector. (b) Schematic illustration of tandem device: starting substrate (left) and final device (right). (c) Depth profile of doping concentration, found using SIMS. (d,e) $30^{\circ}$ tilted SEM image of etched nanowires with radii of $100 \mathrm{~nm}$ and $120 \mathrm{~nm}$. (f) $30^{\circ}$ tilted SEM image of etched mesa structure defining substrate a photodetector. Nanowire array is located in the center. (g,h) Optical microscope images of nanowires (radii: $100 \mathrm{~nm}$ and $120 \mathrm{~nm}$ ) array fabricated on a mesa. Insets are magnified views of nanowire arrays. (i) Wire bonding. Upper right corner is connected to indium tin oxide (ITO). Lower left corner is connected to ITO layer. Substrate contact is not shown in this photo.

We used a multilayered epitaxial wafer that contains $p+/ n-/ n+/ n-/ p+$ layers for device fabrication.(Fig. 1(b)) NW photodetectors are to be fabricated in the top $\mathrm{p}+/ \mathrm{n}-\mathrm{n}+$ layers. The bottom $\mathrm{n}+\mathrm{n}-/ \mathrm{p}+$ layers are for the substrate $\mathrm{p}-\mathrm{i}-\mathrm{n}$ 
junction. Fabrication begins with etching NWs using the method of reference [3]. Scanning electron microscopy (SEM) image of NWs with radii of $100 \mathrm{~nm} / 120 \mathrm{~nm}$ are shown in Fig. 1(d,e). We fabricate arrays of $100 \times 100$ NWs with a pitch of $1 \mu \mathrm{m}$. The NWs have heights of $2.55 \mu \mathrm{m}$. A mesa structure is then etched to define the substrate photodetector. The height of mesa is $8.4 \mu \mathrm{m}$. The lateral extent of substrate photodetector is $2 \mathrm{~mm} \times 2 \mathrm{~mm}$ (Fig. 1(f)). Fig. 1(g,h) show optical microscope images, with the green and orange squares in the centers being the nanowire arrays. We use silver epoxy and gold wires to make electrical contacts to the indium tin oxide (ITO) top layer, the $\mathrm{n}+$ layer, and the substrate (Fig. 1(i)).
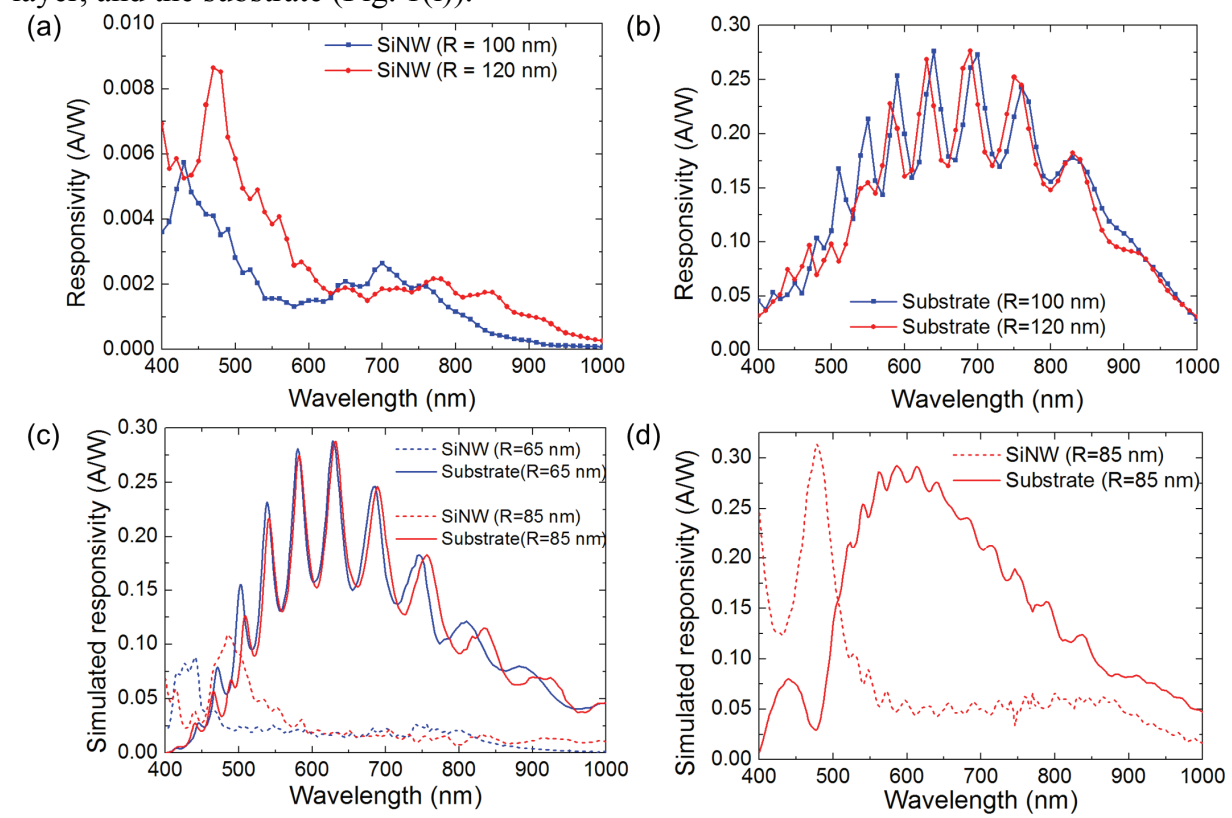

Figure $2(a, b)$ Measured responsivities of nanowire photodetectors(a) and the corresponding substrate photodetectors(b). (c) Simulated responsivities. (d) Simulated responsivities in ideal case (nanowire with radius of $85 \mathrm{~nm}$, height of $4 \mu \mathrm{m}$ tall and pitch of $0.7 \mu \mathrm{m}$ ). ITO layer is removed and thickness of top $\mathrm{n}+$ layer in substrate photodetector is assumed to be $100 \mathrm{~nm}$.

Measured responsivities of the photodetectors comprising NWs with radii (R) of $100 \mathrm{~nm}$ and $120 \mathrm{~nm}$ are found using the method of reference [3] (Fig. 2(a)). The distinct peaks come from the wavelength-dependent field distributions of the guided modes [3]. Fig. 2(b) shows responsivities of the corresponding substrate photodetectors. There are ripples due to the PMMA film (Fig. 1(b)) acting as a Fabry-Perot cavity. Fig. 2(c) shows responsivities simulated using the finite-difference time-domain method. We assume that internal quantum efficiency is $100 \%$. We note that these simulations are for NWs whose radii are $35 \mathrm{~nm}$ smaller than the e-beam lithography design values. The difference in magnitude of responsivity between experiment and simulation may come from recombination at the NW surface. In both measurements and simulations, the responsivity of the second substrate photodetector $(\mathrm{R}=120 \mathrm{~nm})$ is suppressed compared to that of the first substrate photodetector $(\mathrm{R}=100 \mathrm{~nm})$ in the spectral range from $\sim 500 \mathrm{~nm}$ to $\sim 600 \mathrm{~nm}$. This is because the second NW array $(\mathrm{R}=120 \mathrm{~nm})$ has strong absorption there, reducing light transmission to the substrate. This shows the influence of NW absorption on the responsivity of substrate photodetector, i.e. our NW arrays are acting as optical filters. However, the filtering effect is not as strong as it could be because of the NW absorption is relatively small. Therefore, we simulate a modified design intended to increase NW absorption. We elongate the NW length from $2.55 \mu \mathrm{m}$ to $4 \mu \mathrm{m}$. The pitch of array is reduced to 0.7 $\mu \mathrm{m}$. Stronger peaks appear in the NW simulated responsivity and stronger dips appear in the substrate's responsivity (Fig. 2(d)). The spectral response of the NW photodetector is therefore analogous to an image sensor containing a $\mathrm{red} / \mathrm{green} / \mathrm{blue}$ filter and the spectral response of the substrate photodetector is analogous to an image sensor containing a cyan/magenta/yellow filter. We believe this approach could permit very efficient image sensors.

\section{References}

[1] L. Cao, J. S. White, J.-S. Park, J. A. Schuller, B. M. Clemens and M. L. Brongersma, "Engineering light absorption in semiconductor nanowire devices," Nat. Mater. 8, 643-647 (2009).

[2] K. Seo, M. Wober, P. Steinvurzel, E. Schonbrun, Y. Dan, T. Ellenbogen and K. B. Crozier, "Multicolored Vertical Silicon Nanowires," Nano Lett. 11, 1851-1856 (2011).

[3] H. Park, Y. Dan, K. Seo, Y. J. Yu, P. K. Duane, M. Wober and K. B. Crozier, "Filter-free image sensor pixels comprising silicon nanowires with selective color absorption," Nano Lett. 14, 1084-1089 (2014). 